OPEN ACCESS

Edited by:

Tao Huang,

Shanghai Institute of Nutrition and Health Chinese Academy of

Sciences (CAS), China

Reviewed by:

Runhua Tian,

The Affiliated Hospital of Qingdao

University, China

Chang Chen,

Tongji University, China

*Correspondence:

Jian Suo

suojian@jlu.edu.cn

Specialty section

This article was submitted to

$R N A$,

a section of the journal

Frontiers in Genetics

Received: 01 April 2021

Accepted: 12 May 2021

Published: 17 June 2021

Citation:

Liu B, Zhang Y and Suo J (2021) Increased Expression of PDK4 Was

Displayed in Gastric Cancer and Exhibited an Association With

Glucose Metabolism.

Front. Genet. 12:689585.

doi: 10.3389/fgene.2021.689585

\section{Increased Expression of PDK4 Was Displayed in Gastric Cancer and Exhibited an Association With Glucose Metabolism}

\author{
Bin Liu, Yang Zhang and Jian Suo* \\ Department of Gastrocolorectal Surgery, The First Hospital of Jilin University, Changchun, China
}

Previous studies reported that pyruvate dehydrogenase kinase 4 (PDK4) is closely related to diabetes, heart disease, and carcinomas. Nevertheless, the role of PDK4 in gastric cancer (GC) occurrence and development is yet poorly understood. Our experiments were taken to evaluate PDK4's function in GC. The Cancer Genome Atlas tumor genome map database was employed to validate the levels of PDK family in different grades and stages of GC. The survival ratio of PDK families in GC was detected by the Kaplan-Meier plotter database. The links existing in the expression of PDK family and the level of tumor-infiltrating immune cells were investigated by tumor immunity assessment resource (TIMER). PDK4-associated signal pathways in GC were analyzed by the Kyoto Encyclopedia of Genes and Genomes pathway analysis. PDK4 mRNA level in the GC cells was measured by qRT-PCR. Cell counting kit-8 and Transwell assays were separately carried out to evaluate PDK4-induced influence on GC cell proliferation, migration, and invasion. Our data suggested that GC cells highly expressed PDK4, and PDK4 expression presented a significant relation with the staging, grade, and survival rate of GC. PDK4 expression presented a positive correlation with the types of different infiltrating immune cells, comprising B cells, $\mathrm{CD} 4^{+} \mathrm{T}$ cells, and dendritic cells. Meanwhile, PDK4 expression exhibited a strong association with macrophages. Survival analysis revealed that the expression of PDK4 displayed a relationship with the prognosis of patients. Therefore, PDK4 was liable to be a biomarker for prognosis. Our results further displayed that PDK4 might modulate the glycolysis level in GC cells, and its expression was associated with GC cell proliferation, migration, and invasion. These data may provide insights into designing a new treatment strategy for GC.

Keywords: PDK4, gastric cancer, glucose metabolism, tumor-infiltrating immune cells, prognosis, biomarker

\section{INTRODUCTION}

Gastric cancer (GC) deriving from the gastric mucosa is one of the deadliest occurring malignancies which threaten patients' life worldwide (Gu et al., 2020a). As the third largest inducer of global carcinoma deaths, GC has brought a huge burden on public health (Graham, 2015; Ishaq and Nunn, 2015). The metastasis of GC is the main reason affecting the patients (Van Cutsem et al., 2016), and the development of carcinoma is closely related to the energy supply. Tumor cells primarily 
acquired energy by glycolysis, which resulted in a great quantity of lactic acid and a small amount ATP, distinguishing from oxidative phosphorylation occurring in the mitochondria of normal cells (Zheng, 2012). Later, the phenomenon of neoplasm uptake energy was defined as Warburg effect, usually arising in numerous tissues of the neoplasm and promoting tumor cell proliferation, invasion, and metastasis (Pelicano et al., 2006; Chen et al., 2007). Several reports have displayed that the Warburg effect, along with its dependence on tumor cells, relied on the intracellular and extracellular environment (Spencer and Stanton, 2019; Yang and Li, 2019). The levels of lactic acid, glycolytic enzymes, and hypoxia-inducible factor-1 are closely related to tumor proliferation and metastasis (Zhong et al., 1999; Lincet and Icard, 2015). What is more, glycolysis could give rise to lactic acid, and the accumulation of lactic acid would generate an acidic microenvironment instead. Under acidic conditions, the extracellular matrix was extremely unstable, and thus this boosted cancer cell metastasis (Dhup et al., 2012). Therefore, targeting the metabolism of cancer cells was a feasible root to ameliorate and unearth new anti-cancer strategies.

The change in aerobic glycolysis is a recognized feature of energy metabolism in cancer cells and is called the Warburg effect. Increased glycolysis is the main energy source for cancer cells to use this metabolic pathway to produce ATP. The glycolytic pathway is regarded as the target of cancer treatment. Cancer cells maintain a high rate of glycolysis. Pyruvate dehydrogenase kinase (PDK) contributes to this phenomenon, which is conducive to apoptosis resistance and cell transformation. Pyruvate dehydrogenase (PDH) was the key executor in facilitating pyruvate entering the tricarboxylic acid cycle. The activity of PDH could be inhibited by PDK, which was responsible for the conversion from mitochondrial oxidation to cytoplasmic glycolysis (Sutendra and Michelakis, 2013). Dichloroacetate (DCA) was an inhibitor of PDK and could alter the metabolism in the opposite direction (Michelakis et al., 2008). DCA could induce apoptosis but hinder tumor growth and decrease the level of HIF1A controlling the response of hypoxia (Sutendra et al., 2013). PDK1, PDK2, PDK3, and PDK4 are four human kinases of the PDK family (Cesi et al., 2017). PDK4 was reported to display an inhibition of pyruvate oxidation and intervene the change from glucose metabolism to fatty acid metabolism (Liu et al., 2017; Pettersen et al., 2019). Previously, some reports revealed that PDK4 have shown a close association with diabetes, heart disease, and carcinomas (Holness et al., 2002; Furuyama et al., 2003). Moreover, PDK4 was also decreased in multiple carcinomas as previously described, such as prostate carcinoma, breast carcinoma, lung carcinoma, and liver carcinoma (Grassian et al., 2011; Mengual et al., 2014; Sun et al., 2014; Choiniere et al., 2017). Besides this, PDK4 could contribute to the inhibition of cell proliferation and induction of apoptosis in lung and breast carcinoma (Grassian et al., 2011; Li et al., 2017). Additionally, the absence of PDK4 could motivate the EMT program and facilitate ovarian carcinoma cell migration and invasion (Sun S. et al., 2017). Nevertheless, the function of PDK4 in GC was yet elusive.

Here the correlation between PDK family and clinicopathological characteristics and prognosis was analyzed by sequencing data sets. The Kyoto Encyclopedia of Genes and Genomes (KEGG) and The Cancer Genome Atlas (TCGA) data revealed the possible molecular functions of PDK4. Besides that, the association existing in PDK4 expression and tumorinfiltrating immune cells was evaluated. We decreased PDK4 expression in GC cells and then validated the impacts on glycolysis. Moreover, our data revealed that an increase in the expression of PDK4 was displayed in GC, and a high level of PDK4 would lead to a lower rate of overall survival (OS) and a higher rate of recurrence. The inhibited PDK4 greatly reduced GC cell proliferation, migration, and invasion.

\section{MATERIALS AND METHODS}

\section{Public Database}

We collected mRNA expression profiles from TCGA GC cohort ${ }^{1}$ and downloaded clinical information from the TCGA data portal (Gu et al., 2021b). The expression level of the PDK family in GC was determined by utilizing the tumor immunity assessment resource (TIMER) ${ }^{2}$ and the TCGA databases.

\section{Analysis of PDK Family and Clinicopathological Characteristic}

The clinicopathological data of GC patients was downloaded from TCGA. We selected GC tissue samples with clinical pathological data, including classification, staging, and depth of invasion, for further analysis. The association existing in PDK family expression levels and clinicopathological value was analyzed by chi-square test.

\section{Analysis of OS}

The Gene Expression Profile Interactive Analysis (GEPIA) ${ }^{3}$ was employed to analyze the data from TCGA database and the expression of genotype in tissues ( $\mathrm{Gu}$ et al., 2020b). KaplanMeier plotter online database ${ }^{4}$ could validate the impact of each gene on the survival rate of GC patients. GEPIA and Kaplan-Meier plotter online databases were both taken to evaluate the prognostic value of PDK4 in patients with GC. The inspection probe number used by PKD4 was 205960_at. The $P$-value of log-rank and hazard ratio with $95 \%$ confidence intervals were measured.

\section{GO Annotation and KEGG Pathway Enrichment Analyses}

For exploring PDK4's function, we employed Enrichr database ${ }^{5}$ for Gene Ontology (GO) annotation and KEGG pathway enrichment analysis. Significant statistical difference is indicated as $P<0.05$ (Zhang et al., 2020; Gu et al., 2021a).

\footnotetext{
${ }^{1}$ http://cancergenome.nih.gov/

${ }^{2}$ https://cistrome.shinyapps.io/timer/

${ }^{3}$ http://gepia.cancer-pku.cn/

${ }^{4}$ http://kmplot.com/analysis/

${ }^{5}$ https://maayanlab.cloud/Enrichr/
} 


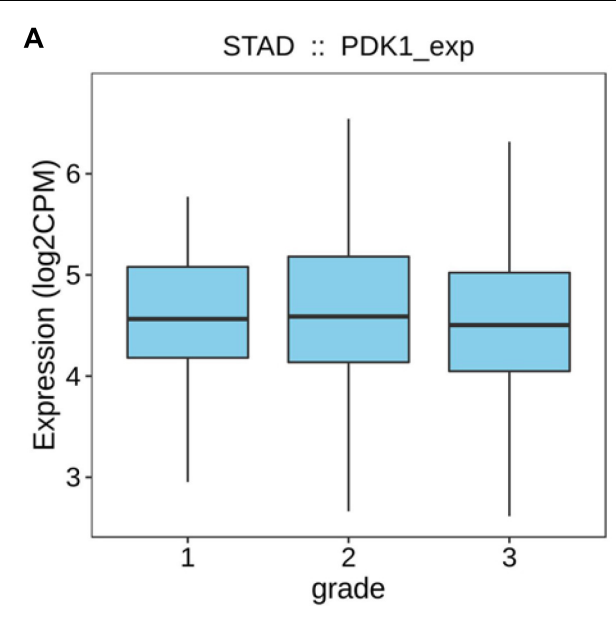

Spearman: rho $=-0.056, p=0.257$

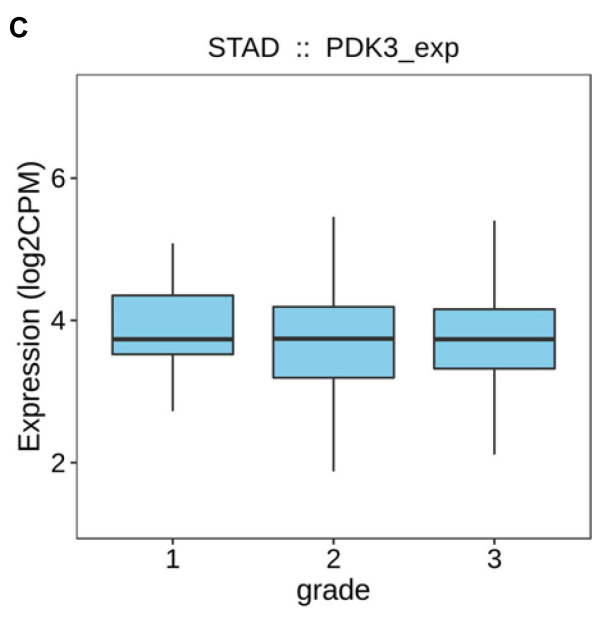

Spearman: $r h o=0.03, p=0.544$

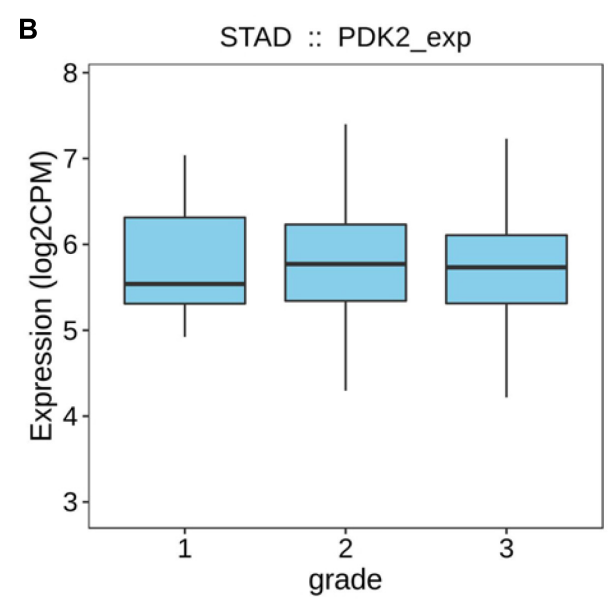

Spearman: $r h o=-0.042, p=0.395$

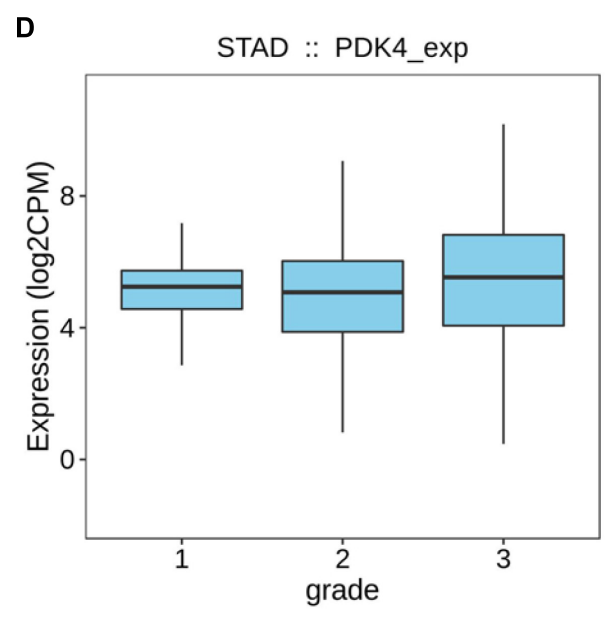

Spearman: $r h o=0.135, p=0.0064$

FIGURE 1 | PDK4 expression exhibited a significant correlation with neoplasm grade of STAD. (A-D) Analysis of the relationship between tumor grade and PDK1, PDK2, PDK3, and PDK4 expression in STAD patients on the basis of The Cancer Genome Atlas database.

\section{Cell Culture}

Normal human gastric epithelium cell line (GES-1) and human GC cells (SGC-7901, MGC-803, HGC 27, and AGS) were acquired from the Cell Bank of Institute of Biochemistry and Cell Biology at the Chinese Academy of Sciences (Shanghai, China). They were maintained in Dulbecco's modified Eagle's containing $10 \%$ fetal bovine serum (FBS) and 1\% P/S (Gibco, United States) under $37^{\circ} \mathrm{C}$ humidified incubator with $5 \% \mathrm{CO}_{2}$.

\section{RNA Extraction and Quantitative Reverse Transcription PCR}

Overall, the RNA of cells was isolated by TRIzol. The extracted RNA was reversely transcribed into cDNA by cDNA synthesis kit (TaKaRa, Japan). Primers designed by Primer 5.0 software were synthesized by Invitrogen. PCR was carried out by utilizing SYBR GREEN on an ABI 7300 plus real-time system. The $2^{-\Delta \Delta C t}$ method was taken to normalize the PDK4 mRNA expression levels in GC cells and control. The RNA primers were as follows: PDK4, 5'-GGAGCATTTCTCGCGCTACA$3^{\prime}$ (forward), 5'-ACAGGCAATTCTTGTCGCAAA-3' (reverse); GAPDH, 5' -CTGGGCTACACTGAGCACC-3' (forward), 5' AAGTGGTCGTTGAGGGCAATG-3' (reverse). GAPDH was used as a reference.

\section{Transient Transfection}

SiRNA (si-PDK4) (100 nM, GenePharma, China) was introduced into AGS cells to silence PDK4, and si-NC was selected as the negative control (NC) for si-PDK4.

Lipofectamine 2000 kit (Invitrogen, Carlsbad, United States) was used for cell transfer. At $48 \mathrm{~h}$ after transfection, different transfected cells were obtained for the next experiment. The sequence of the siRNA is as follows: siPDK41: CGCCAACATTCTGAAGGAAATTGAT; si-NC: UUCUCCGAACGUGUCACGUTT. 


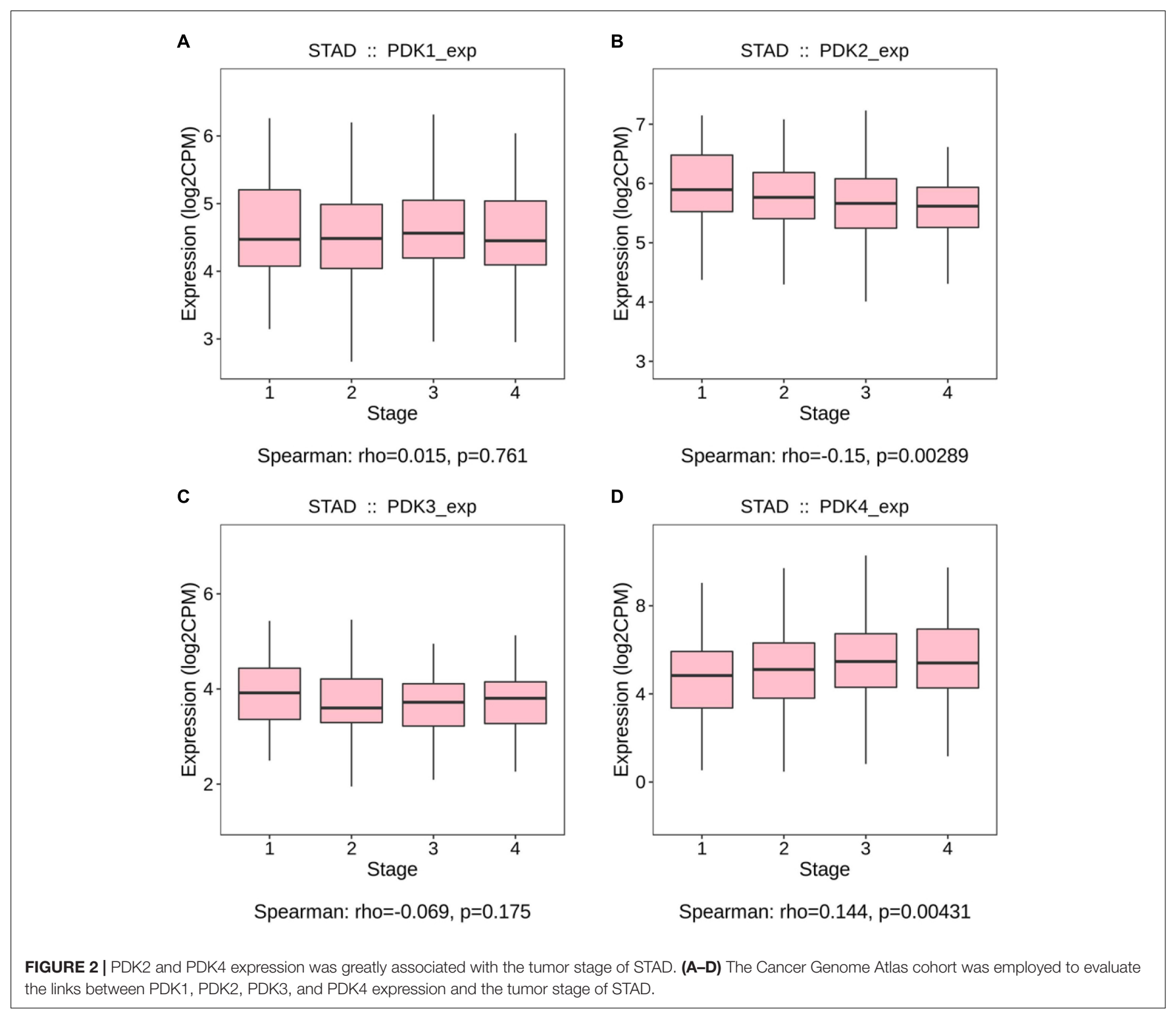

\section{Cell Proliferation Assay}

CCK-8 kit (Dojindo, Japan) was employed to determine the ability of cell proliferation in GC cells. The indicated number of GC cells was plated in a 96-well plate and then subjected to different treatments at the indicated time. The OD value of $450 \mathrm{~nm}$ was detected after incubation with CCK-8 solution on Fluoroskan Ascent Fluorometer (Thermo Fisher Scientific, Finland).

\section{Transwell Assays}

Cell migration and invasion were measured using transwell chamber ( $8-\mu \mathrm{m}$ pore size; Corning Co., Corning, United States) without/with Matrigel (Becton Dickinson, New York, United States). At $48 \mathrm{~h}$ after transfection, cells in $200 \mu \mathrm{l}$ of medium, in the absence of serum, were added into the upper chambers. Then, $600 \mu \mathrm{l}$ of medium containing 10\% FBS was added into the lower chambers. At $24 \mathrm{~h}$ post-incubation, a cotton-tipped swab was applied to remove the cells staying in the lower chamber. All the cells removed were fixed by methanol for $10 \mathrm{~min}$ and stained with DAPI for $30 \mathrm{~min}$ at room temperature and protected from light. The number of cells was calculated under an inverted phase-contrast microscope (Olympus, Tokyo, Japan) (Kunig et al., 2020).

\section{Statistical Analysis}

All derived data were shown as the mean \pm SEM from three separate experiments in triplicate. GraphPad Prism 5 software (GraphPad Software, Inc.) was carried out to perform data analysis. Student's $t$-test was conducted to perform comparisons between the two groups. A comparison amid three groups was evaluated by one-way ANOVA (Shi et al., 2020), subsequently subjecting to Kaplan-Meier method with log-rank test to test the survival curves (Sun Y. et al., 2017; Gu et al., 2018). Significant 
A

STAD :: PDK1_exp

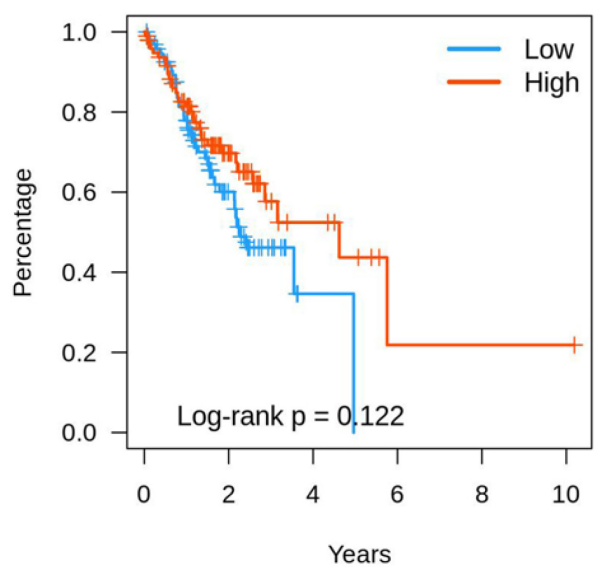

C

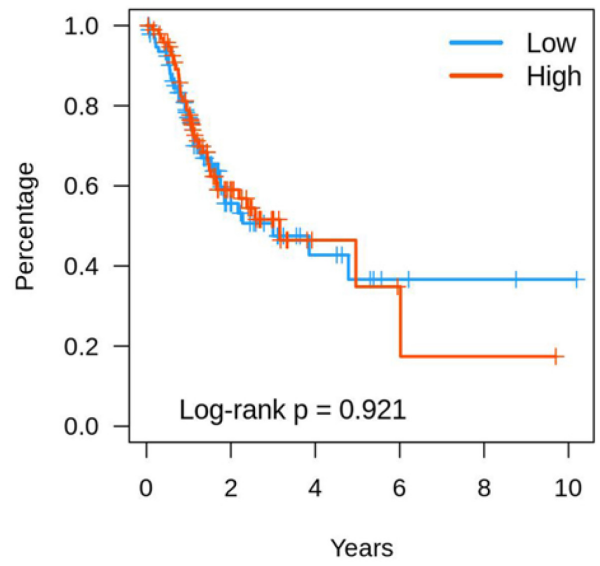

B

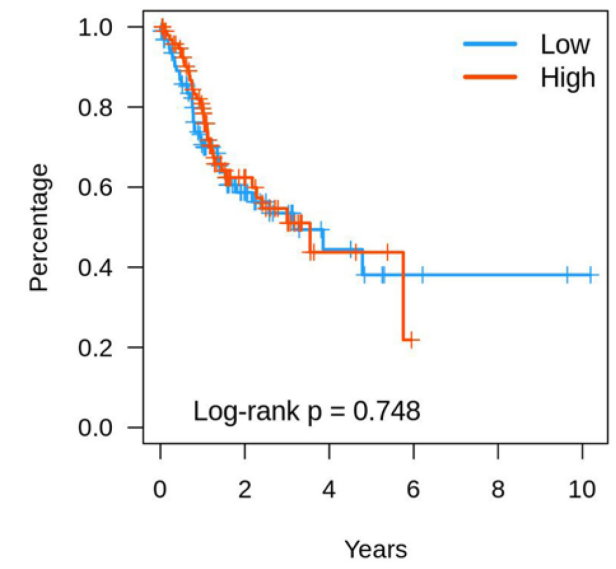

D

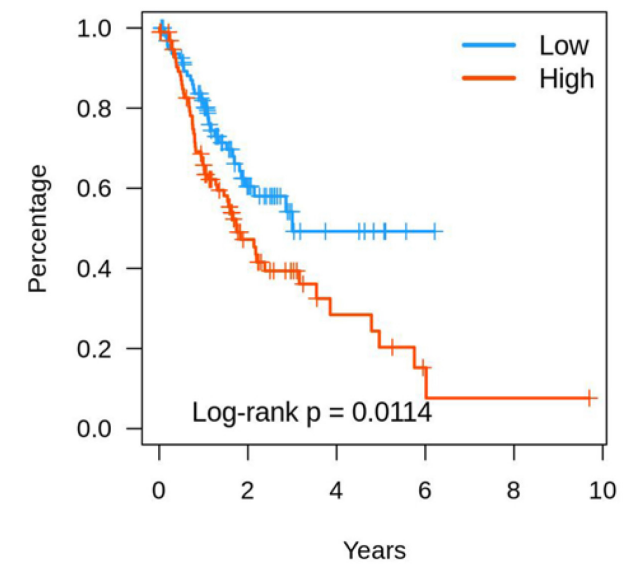

FIGURE 3 | A high expression of PDK4 indicated a lower survival rate. (A-D) Kaplan-Meier analysis of the association between PDK1, PDK2, PDK3, and PDK4 expression and the survival rate of STAD patients.

statistical difference is indicated as $P<0.05$ (Chen et al., 2020, 2021).

\section{RESULTS}

\section{PDK Family and Clinicopathological Parameters}

The links between the expression of PDK family and clinicopathological parameters, including the grade and the stage of tumor, were analyzed on the basis of the clinical and pathological data of STAD patients. No significant statistical relationship existed between PDK1, PDK2, and PDK3 expression and tumor grade $(P=0.257, P=0.395, P=0.544$, Figures 1A-C). Importantly, PDK4 expression exhibited a large association with the grade of tumor $(P=0.0064$, Figure 1D). In grade 3 , the expression level of PDK4 was the highest. Similarly, no significant relationship was shown between PDK1 and PDK3 expression and tumor stage $(P=0.761, P=0.175$, Figures 2A,C). PDK2 and PDK4 expression had shown a great correlation with tumor stage $(P=0.00289, P=0.00431$, Figures 2B,D). Interestingly, the expression of PDK2 was highest in stage 1 , while the expression of PDK4 was lowest in stage 1.

\section{Identification of Prognosis of PDK Family}

Kaplan-Meier analysis was used to identify the prognosis parameter of PDK1, PDK2, PDK3, and PDK4. Our data revealed that PDK1, PDK2, and PDK3 expression had no significant relationship with the STAD patients' survival rate (Figures 3AC). Interestingly, the PDK4 expression was evidently related with survival rate (Figure 3D). Further data revealed that a lower survival rate was demonstrated in STAD patients with a high expression of PDK4. According to the survival analysis, the higher the expression of PDK4, the higher the probability of a poorly prognostic status. 

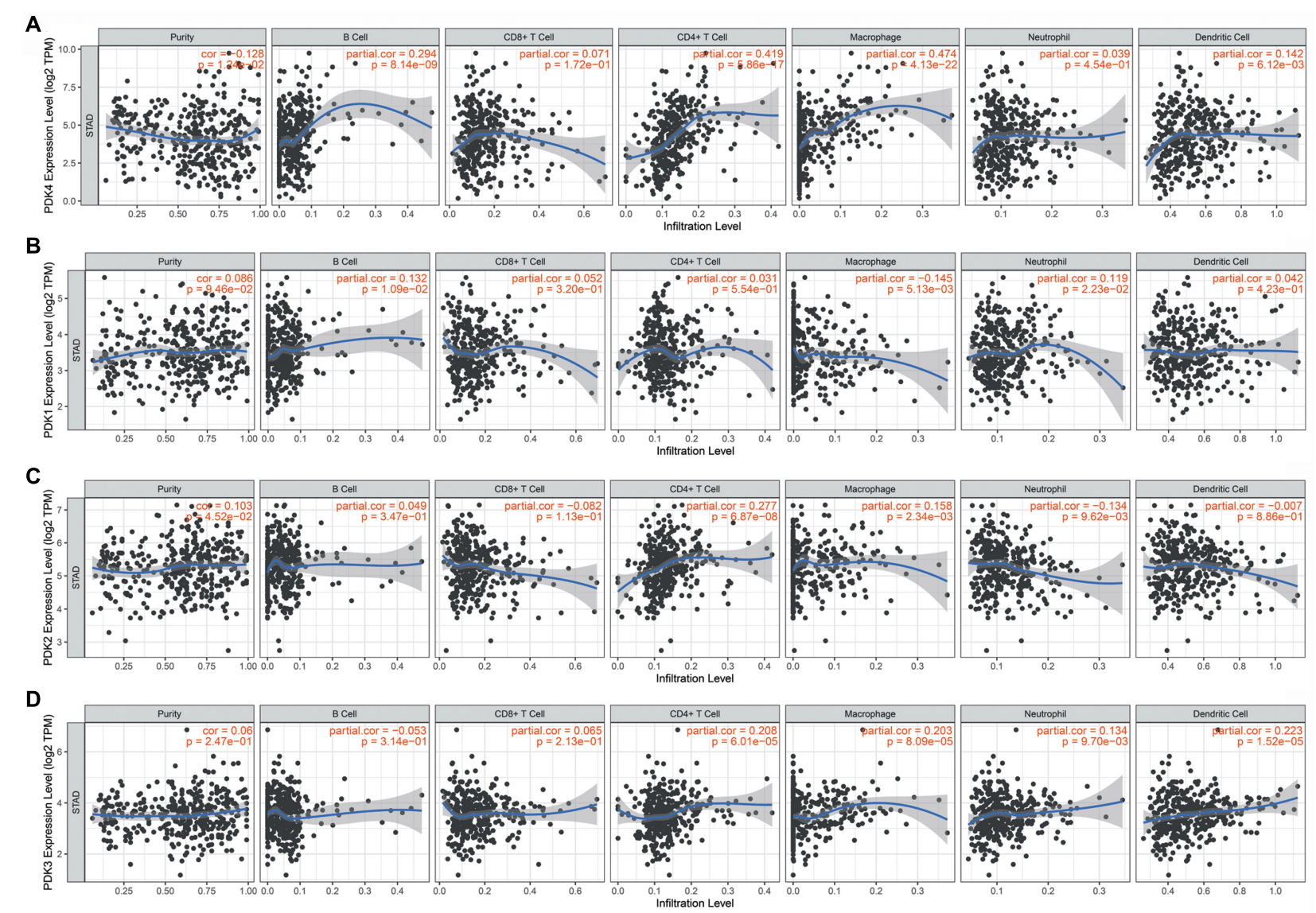

FIGURE 4 | PDK4 was significantly associated with tumor-infiltrating immune cells in gastric cancer (GC). Obvious association of the PDK family with the level of tumor immune filtration in GC. PDK4 (A), PDK1 (B), PDK2 (C), and PDK3 (D).

\section{PDK4 Significantly Correlated With Tumor-Infiltrating Immune Cells in GC}

The hidden links between PDK1, PDK2, PDK3, and PDK4 expression and tumor-infiltrating immune cells in GC were assessed by the TIMER database. The data suggested that PDK4 expression had positive links with different infiltrating immune cells level, comprising B cells $(r=0.294, P=8.14 \mathrm{e}-$ $09), \mathrm{CD}^{+} \mathrm{T}$ cells $(r=0.419, P=5.86 \mathrm{e}-17)$, and dendritic cells $(r=0.142, P=6.12 \mathrm{e}-03)$ and exhibited a strong correlation with macrophages $(r=0.474, P=4.13 \mathrm{e}-22)$ (Figure 4A). At the same time, our results showed that PDK1 (Figure 4B), PDK2 (Figure 4C), and PDK3 (Figure 4D) were not significantly related to immune cells. Collectively, our data suggested that PDK4, along with the co-expressed genes, probably participated in immune response in the microenvironment of tumor via exerting an effect on immune cells, especially macrophages.

\section{PDK4 Was an Adverse Factor for GC Prognosis}

The prognostic parameter of PDK4 in GC was analyzed by utilizing GEPIA data and the Kaplan-Meier plotter database accompanied by GEO data. The results showed that, compared with gastric cancer patients with low PDK4 expression, the OS of gastric cancer patients with high PDK4 expression had a significantly lower first progression survival and post-progression survival $(P=0.00036, P=0.014, P=5.8 \mathrm{e}-05$ (Figures 5AC). Compared with patients with gastric cancer and low PDK4 expression, patients with stage 1,2 , and 3 gastric cancer with high PDK4 expression had a significantly lower OS $(P=0.012$, $P=0.0018, P=0.00053, P=0.21$; Figures 5D-G). Compared with patients with gastric cancer with low PDK4 expression, patients with stage N0 gastric cancer with high PDK4 expression had a significantly lower OS (Figure 5H, $P=0.0059$ ). Compared with patients with gastric cancer with low PDK4 expression, the OS of $\mathrm{N} 1+\mathrm{N} 2+\mathrm{N} 3$ gastric cancer patients with high PDK4 expression was significantly lower $(P=1.1 \mathrm{e}-05$, Figure 5I). In summary, we infer that the high expression of PDK4 is related to the poor prognosis of gastric cancer.

\section{Functional Analysis of PDK4}

KEGG analysis showed that cGMP-PKG signaling pathway, cell cycle, DNA replication, dilated cardiomyopathy, extracellular membrane-receptor interaction, adrenergic signaling in cardiomyocytes, circadian entrainment, vascular smooth muscle 

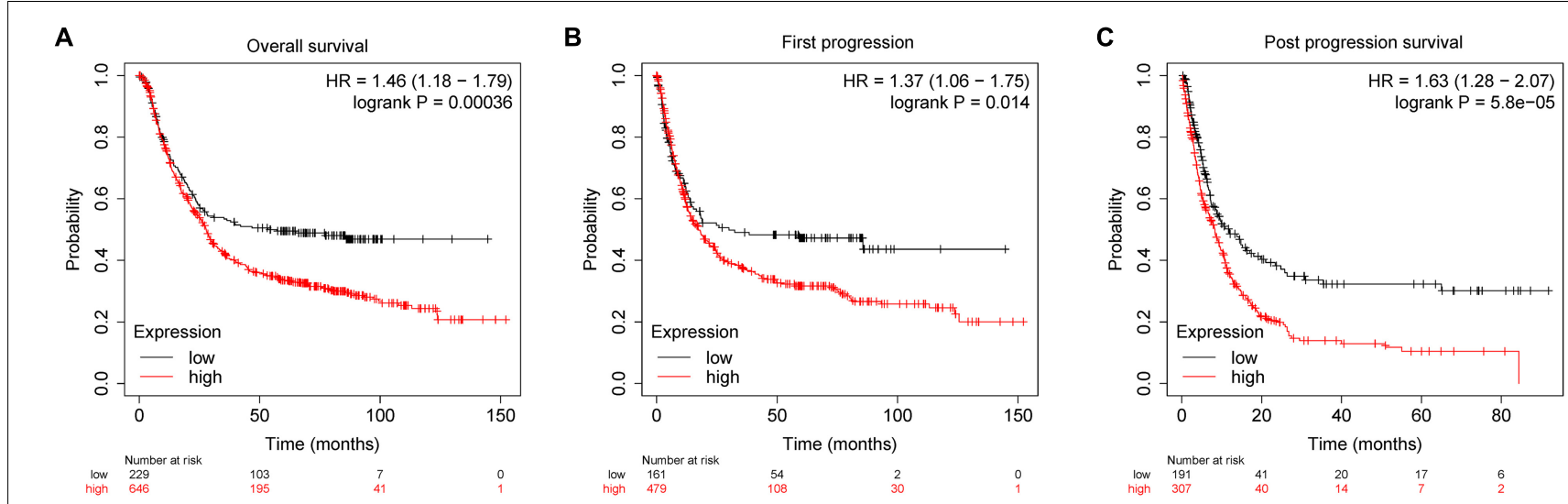

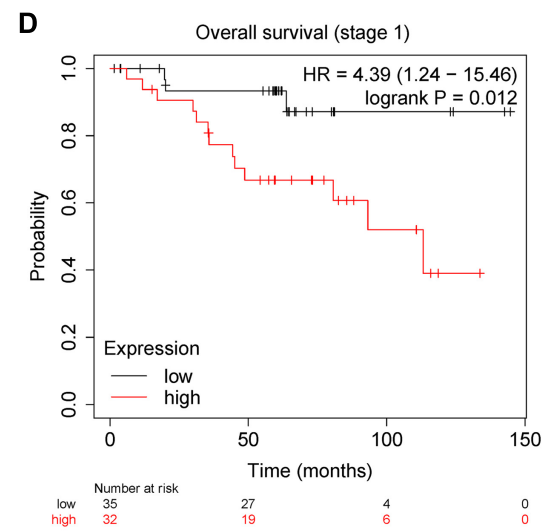

E

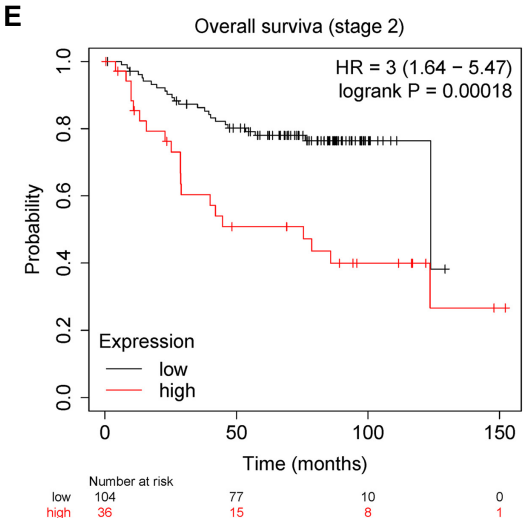

$\mathbf{H}$

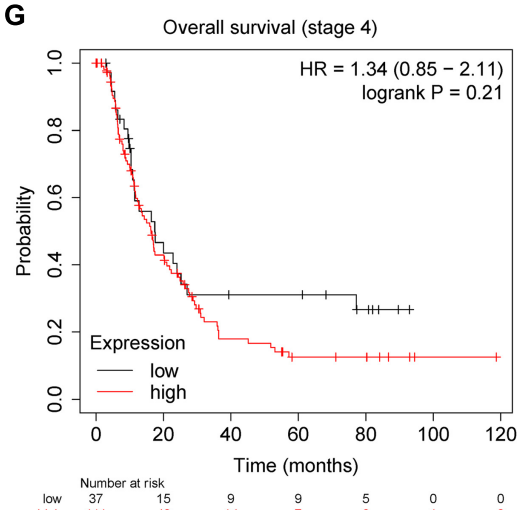

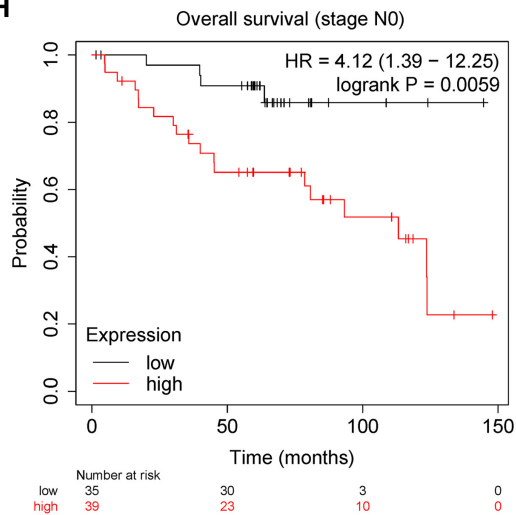

$\mathbf{F}$

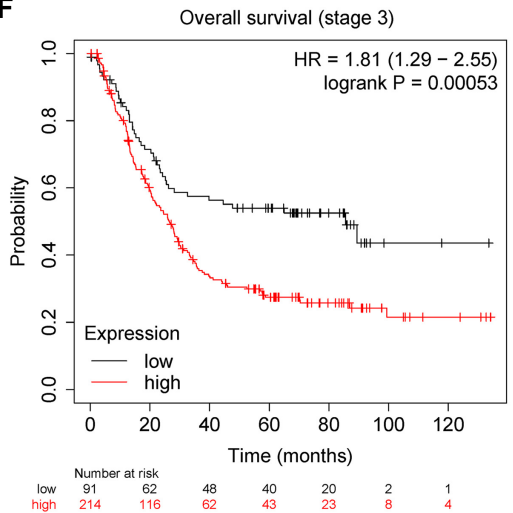

I

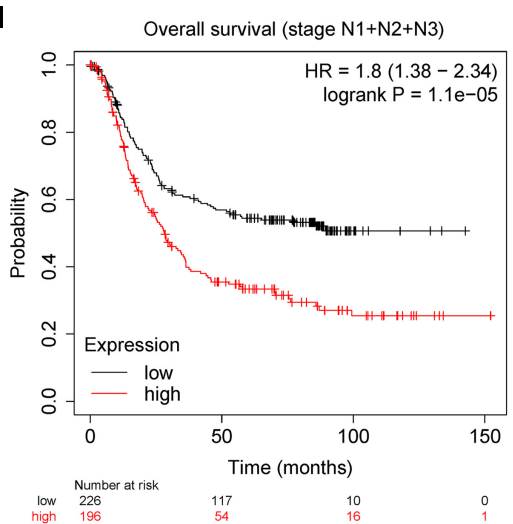

FIGURE 5 | PDK4 was significantly related to the survival of gastric cancer (GC) patients. Kaplan-Meier plotter was used to analyze the relationship between the expression level of PDK4 and the overall survival. (A) First progression, (B) post-progression survival, (C) post-progression survival, (D) overall survival (stage 1), (E) overall survival (stage 2), (F) overall survival (stage 3), (G) overall survival (stage 4), (H) overall survival (stage N0), and (I) overall survival (stages N1 + N2 + N3) of gastric cancer patients.

contraction, calcium signaling pathway, and progesteronemediated oocyte maturation were the most important pathways (Figure 6A). GO analysis data revealed that PDK4 primarily took part in the positive modulation of DNA replication (GO:0006260), nervous system development (GO:0007399), DNA-dependent DNA replication (GO:0006261), regulation of heart contraction (GO:0008016), muscle contraction (GO:0006936), regulation of cardiac conduction (G0:1903779), membrane depolarization during cardiac muscle cell action potential (GO:0086012), mitotic spindle organization (GO:0007052), cAMP-mediated signaling (GO:0019933), and muscle organ development (GO:0007517) (Figure 6B).

\section{Highly Expressed PDK4 mRNA Was Observed in GC Cell}

In order to verify whether the expression of PDK4 in GC cell lines was also upregulated, we also conducted qRT-PCR 


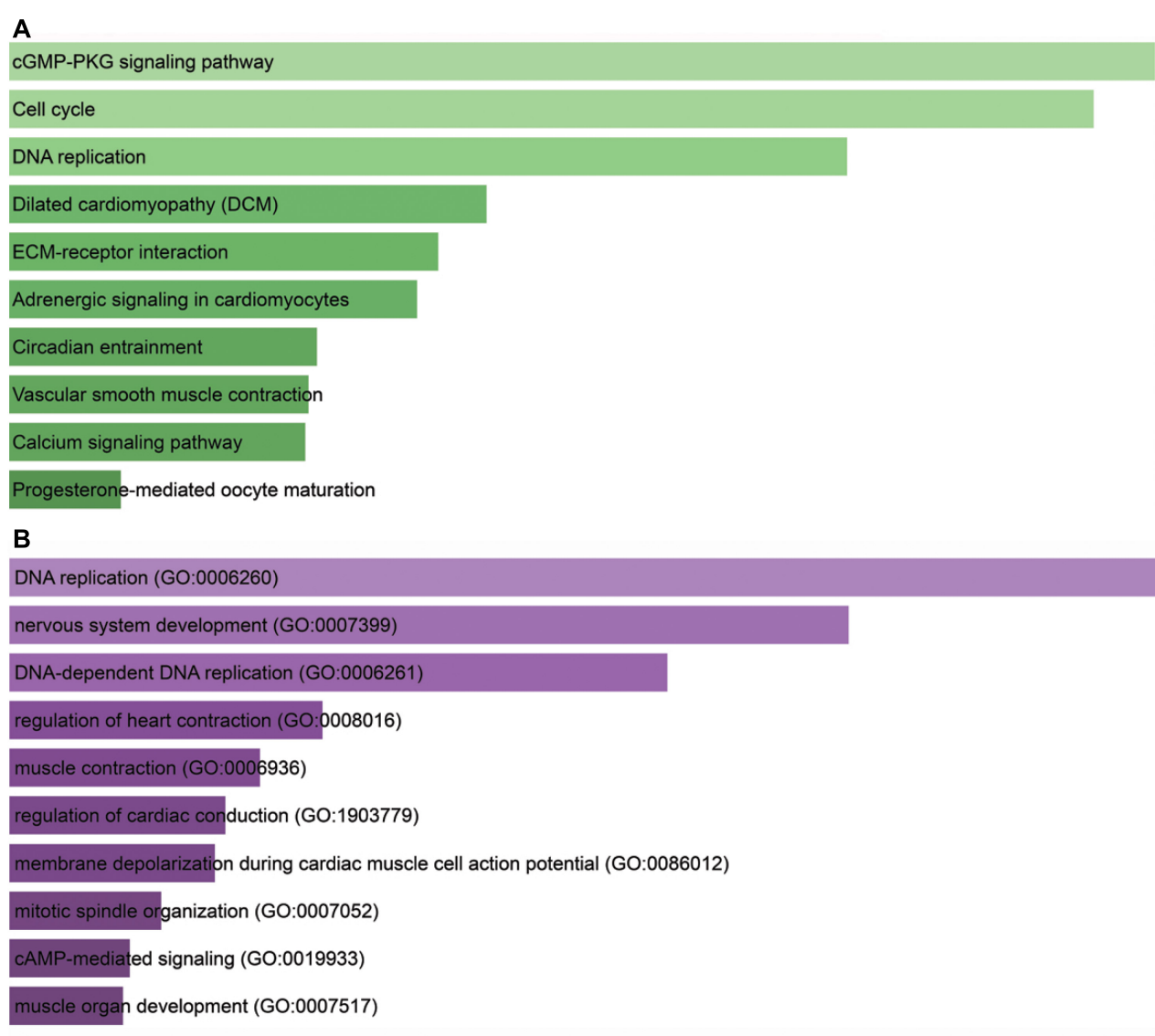

FIGURE 6 | Bioinformatics analysis for PDK4. (A) Kyoto Encyclopedia of Genes and Genomes analysis of enriched pathways of PDK4. (B) Gene Ontology analysis of PDK4. The X-axis indicated gene count; the Y-axis meant enriched pathway or GO term. The color represents the $P$-value.
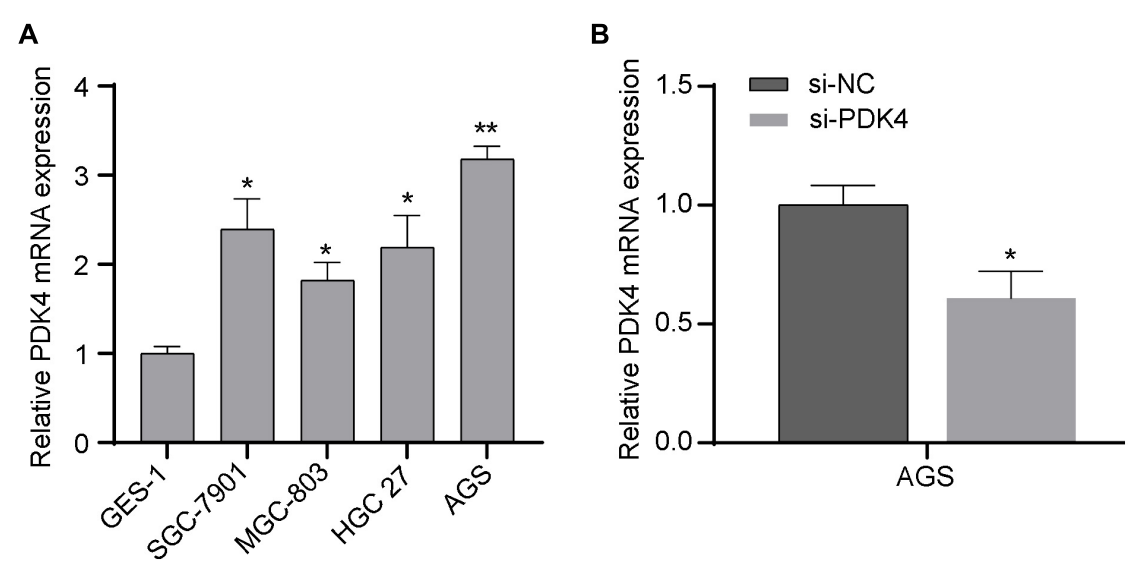

FIGURE 7 | Upregulated PDK4 was shown in gastric cancer (GC) cells, and the si-PDK4 knockdown efficiency was high. (A) qRT-PCR analysis of PDK4 expression in four GC cell lines and normal cell lines. (B) qRT-PCR analysis of PDK4 expression in si-PDK4-transfected AGS cells. ${ }^{*} P<0.05,{ }^{\star \star} P<0.01$.

to measure and compare the PDK4 level in several GC cell lines and human normal gastric cell lines. Our data suggested that PDK4 was significantly upregulated in the following GC cell lines compared to normal cell lines: SGC-7901, MGC-823, HGC 27, and AGS $(P<0.05$, Figure 7A). Our data was in line with the results of the database, that is, the expression of PDK4 in GC was upregulated. In addition, we found that relative to the si-NC-transfected group, the PDK4 expression level in the si-PDK4-transfected AGS cells was reduced $(P<0.05$, Figure 7B). 
A

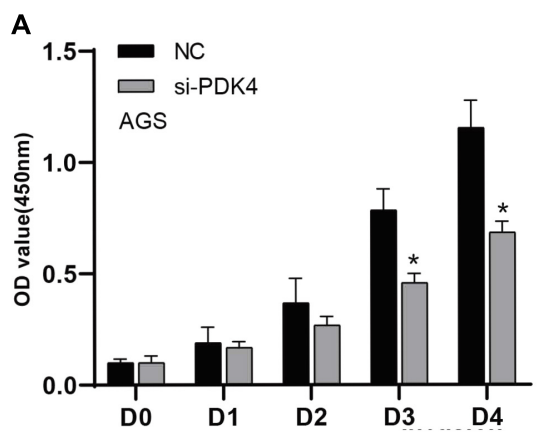

C

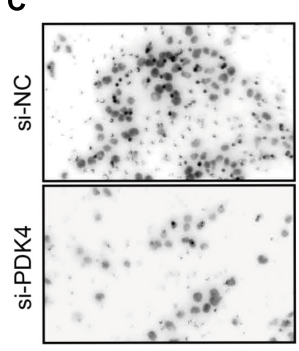

B
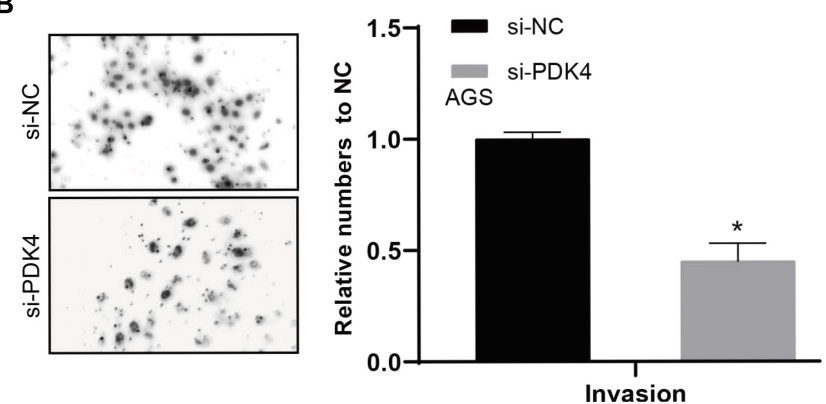

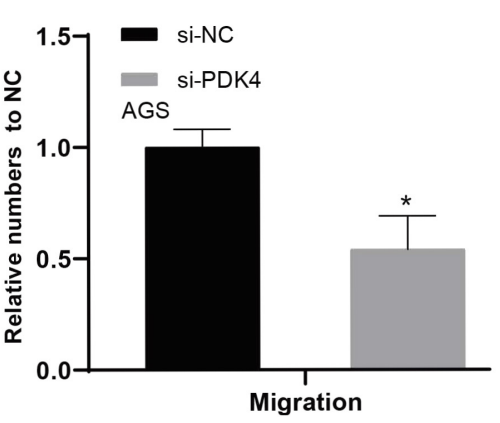

FIGURE 8 | siRNA-mediated silencing of PDK4 expression inhibits the proliferation, invasion, and migration of STAD cells. (A-C) Assessment of the capability of cell proliferation, cell invasion, and cell migration in si-PDK4-transfected AGS cells. ${ }^{*} P<0.05$.

\section{PDK4 Expression Exerted an Effect on GC Cell Proliferation, Invasion, and Migration}

The CCK-8 analysis data revealed that silencing the PDK4 expression contributed to inhibited GC cell proliferation. Relative to the si-NC-treated group, the si-PDK4-transfected AGS cells showed reduced proliferation ability $(P<0.05$, Figure $8 \mathbf{A})$. In order to further study the correlation between the low PDK4 expression and the invasion and migration of GC cells, we conducted Transwell assay. Our data suggested that the cell number of invasion $(P<0.05$, Figure 8B) and migration $(P<0.05$, Figure 8C) in the si-PDK4-transfected AGS cells was fewer than that in the si-NC-transfected group.

\section{DISCUSSION}

Currently, GC has become a global issue concerning human health. The study of GC never stops. PDK4 was reported to act as an essential mediator of cell metabolism and to display importance in the development of tumorigenesis and metastasis. Many potential targets in cancers were developed and studied ( $\mathrm{Gu}$ and Chen, 2020; Bao et al., 2021). Previous reports had revealed that upregulating PDK4 could affect numerous carcinomas, such as lung carcinoma, breast carcinoma, ovarian carcinoma, and colon carcinoma (Yang et al., 2019). Overexpression of PDK4 also brought about drug resistance, survival, and metastasis (Wang et al., 2019). A new study suggested that PDK4 plays an oncogene role in GC, consistent with our research. Here our results implied that the highly expressed PDK4 mRNA was observed in the cell lines of GC, suggesting that PDK4 might promote GC proliferation. In addition, we validated the association between the expression of PDK4 and clinicopathological characteristics, including the prognosis and survival rate of patients, by analyzing the clinical samples of GC. The data indicated that PDK4 might be regarded as a newly generated indicator for GC patients' prognosis. TCGA data-based pathway enrichment and gene correlation analysis revealed that GC cells might enrich PDK4 via glycolysisrelated pathways.

It is known that changes in energy metabolism functions importantly in carcinoma progression. The capability of accelerating the uptake and the oxidation of glucose is characteristic of most malignant neoplasms. Most cancer cells prefer glycolysis rather than mitochondrial oxidative phosphorylation (OXPHOS). Glycolysis is the primary energy supply pathway for the rapid proliferation of tumor cells, allowing them to adapt to the hypoxic environment and further raise their malignant potential (Chen et al., 2007; Zheng, 2012). In humans, PDK has four isoforms (1-4). According to reports, SPRY4-IT1 promotes the survival of colorectal cancer cells by regulating PDK1-mediated glycolysis. PDK2enhanced glycolysis promotes fibroblast proliferation in thyroidassociated ophthalmopathy. In the study of chemoresistance in gastric cancer, PDK3 is highly expressed to promote glycolysis in chemoresistant cancer cells. PDK4 was shown to facilitate the glycolysis of aerobic and cell proliferation of neoplasms 
(Yu et al., 2017). Studies have shown that $\mathrm{m}^{6} \mathrm{~A}$ regulates the glycolysis of cancer cells through PDK4 (Li et al., 2020). We assumed that it may result from the fact that PDK4 reduced the content of acetyl CoA, a precursor of the synthesis of fatty acid and the production of energy. Nevertheless, tumor cells were engaged in a sole metabolic reprogramming, which was identified as the Warburg effect (Chen et al., 2007). Thus, the content of acetyl CoA was not essential. Therefore, PDK4 acts as a balancer between glycolysis and oxidative phosphorylation. Nevertheless, the particular mechanism relating to PDK4 in carcinoma still needed to be explored. PDK4 acts as an important gene relating to glucose metabolism and participates in the control of glucose metabolism and mitochondrial respiration (Guda et al., 2018). Therefore, we want to know whether PDK4 affects the proliferation and invasion of GC via modulating glycolysis. Our research shows that, on the basis of TCGA data for pathway enrichment and gene correlation analysis, PDK4 is usually reduced in the clinical samples of GC. GC may enrich PDK4 through glycolysis-related pathways, thereby affecting the progression of GC. Additionally, the function of PDK4, which was involved in glycolysis, was identified through gene set enrichment analysis.

Most studies focused on PDK1, PDK2, or PDK3. Zhang et al. (2006) found that the most frequently occurring isoforms of PDK were PDK2 and PDK4. However, PDK4 was not widely validated in its transformation. For exploring the functions and prognostic implication of PDK4 in GC, we conducted conclusive experiments. To evaluate the role of PDK4 in tumorigenesis, we ablated PDK4 expression by siRNAs in human GC cells. Our data indicated that a high expression of PDK4 brought about a short OS rate of patients with GC. Knockdown of PDK4 attenuated the ability of GC cell proliferation, invasion, and metastasis in vitro.

\section{REFERENCES}

Bao, Y., Gu, C., Xie, H., Zhao, S., Xie, D., Chen, C., et al. (2021). Comprehensive study of neoadjuvant targeted therapy for resectable non-small cell lung cancer. Ann. Transl. Med. 9:493.

Cesi, G., Walbrecq, G., Zimmer, A., Kreis, S., and Haan, C. (2017). ROS production induced by BRAF inhibitor treatment rewires metabolic processes affecting cell growth of melanoma cells. Mol. Cancer 16:102.

Chen, C., Zhang, X., Gu, C., Wang, Y., Liu, K., Pan, X., et al. (2020). Surgery performed at night by continuously working surgeons contributes to a higher incidence of intraoperative complications in video-assisted thoracoscopic pulmonary resection: a large monocentric retrospective study. Eur. J. Cardiothorac. Surg. 57, 447-454.

Chen, J., Gu, C., Chen, X., Dai, C., Zhao, S., Xie, H., et al. (2021). Clinicopathological and prognostic analyses of 86 resected pulmonary lymphoepithelioma-like carcinomas. J. Surg. Oncol. 123, 544-552. doi: 10.1002/ jso. 26276

Chen, Z., Lu, W., Garcia-Prieto, C., and Huang, P. (2007). The Warburg effect and its cancer therapeutic implications. J. Bioenerg. Biomembr. 39, 267-274. doi: 10.1007/s10863-007-9086-x

Choiniere, J., Wu, J., and Wang, L. (2017). Pyruvate dehydrogenase kinase 4 deficiency results in expedited cellular proliferation through E2F1-mediated increase of cyclins. Mol. Pharmacol. 91, 189-196. doi: 10.1124/mol.116.106757

Dhup, S., Dadhich, R. K., Porporato, P. E., and Sonveaux, P. (2012). Multiple biological activities of lactic acid in cancer: influences on tumor growth, angiogenesis and metastasis. Curr. Pharm. Des. 18, 1319-1330. doi: 10.2174/ 138161212799504902
Additionally, due to the increased expression in gastric cancer, PDK4 was a probable target for the treatment of GC.

This study has some limitations. First, it is necessary to verify the regulation of PDK4 glycolysis in GC cells. Second, the protein expression level of PDK4 needs to be tested internally in GC clinical samples. In future studies, we will collect more GC clinical samples to detect the protein expression level of PDK4 and its prognostic value. We also plan to further explore the in vivo function of PDK4 in animal models.

Taken together, not only is PDK4 associated with the glycolysis and proliferation of neoplasm but also it exerted an effect on the development and prognosis of neoplasm. Our data displayed that overexpressing PDK4 in GC exhibited an association with clinicopathological parameters and poor prognostic status. Highly expressed PDK4 presented an intimate relation with the level of infiltrating immune cells. Our findings demonstrated that PDK4 was a biomarker for GC prognosis and a promising target in the therapy of GC.

\section{DATA AVAILABILITY STATEMENT}

The original contributions presented in the study are included in the article/supplementary material, further inquiries can be directed to the corresponding author/s.

\section{AUTHOR CONTRIBUTIONS}

JS designed the study. All authors collected the data, performed the experiments, wrote the manuscript, and approved the final version of the manuscript.

Furuyama, T., Kitayama, K., Yamashita, H., and Mori, N. (2003). Forkhead transcription factor FOXO1 (FKHR)-dependent induction of PDK4 gene expression in skeletal muscle during energy deprivation. Biochem. J. 375( $\mathrm{Pt} 2)$, 365-371. doi: 10.1042/bj20030022

Graham, D. Y. (2015). Helicobacter pylori update: gastric cancer, reliable therapy, and possible benefits. Gastroenterology 148:719-31e3.

Grassian, A. R., Metallo, C. M., Coloff, J. L., Stephanopoulos, G., and Brugge, J. S. (2011). Erk regulation of pyruvate dehydrogenase flux through PDK4 modulates cell proliferation. Genes Dev. 25, 1716-1733. doi: 10.1101/gad. 16771811

Gu, C., and Chen, C. (2020). Methylation in lung cancer: a brief review. Methods Mol. Biol. 2204, 91-97. doi: 10.1007/978-1-0716-0904-0_8

Gu, C., Chen, J., Dang, X., Chen, C., Huang, Z., Shen, W., et al. (2021a). Hippo pathway core genes based prognostic signature and immune infiltration patterns in lung squamous cell carcinoma. Front. Oncol. 11:680918. doi: 10. 3389/fonc. 2021.680918

Gu, C., Huang, Z., Dai, C., Wang, Y., Ren, Y., She, Y., et al. (2018). Prognostic analysis of limited resection versus lobectomy in stage IA small cell lung cancer patients based on the surveillance, epidemiology, and end results registry database. Front. Genet. 9:568. doi: 10.3389/fgene.2018.00568

Gu, C., Shi, X., Dai, C., Shen, F., Rocco, G., Chen, J., et al. (2020a). RNA m6A modification in cancers: molecular mechanisms and potential clinical applications. Innovation 1:100066. doi: 10.1016/j.xinn.2020.100066

Gu, C., Shi, X., Huang, Z., Chen, J., Yang, J., Shi, J., et al. (2020b). A comprehensive study of construction and analysis of competitive endogenous RNA networks in lung adenocarcinoma. Biochim. Biophys. Acta Proteins. Proteom. 1868:140444. doi: 10.1016/j.bbapap.2020.140444 
Gu, C., Shi, X., Qiu, W., Huang, Z., Yu, Y., Shen, F., et al. (2021b). Comprehensive analysis of the prognostic role and mutational characteristics of m6A-related genes in lung squamous cell carcinoma. Front. Cell Dev. Biol. 9:661792. doi: $10.3389 /$ fcell.2021.661792

Guda, M. R., Asuthkar, S., Labak, C. M., Tsung, A. J., Alexandrov, I., Mackenzie, M. J., et al. (2018). Targeting PDK4 inhibits breast cancer metabolism. Am. J. Cancer Res. 8, 1725-1738.

Holness, M. J., Bulmer, K., Gibbons, G. F., and Sugden, M. C. (2002). Up-regulation of pyruvate dehydrogenase kinase isoform 4 (PDK4) protein expression in oxidative skeletal muscle does not require the obligatory participation of peroxisome-proliferator-activated receptor alpha (PPARalpha). Biochem. J. 366(Pt 3), 839-846. doi: 10.1042/bj20020754

Ishaq, S., and Nunn, L. (2015). Helicobacter pylori and gastric cancer: a state of the art review. Gastroenterol. Hepatol. Bed. Bench 8(Suppl. 1), S6-S14.

Kunig, V. B. K., Potowski, M., Akbarzadeh, M., Klika Skopic, M., Dos Santos Smith, D., Arendt, L., et al. (2020). TEAD-YAP interaction inhibitors and MDM2 binders from DNA-encoded indole-focused Ugi peptidomimetics. Angew. Chem. Int. Ed. Engl. 59, 20338-20342. doi: 10.1002/anie.202006280

Li, G., Li, M., Hu, J., Lei, R., Xiong, H., Ji, H., et al. (2017). The microRNA-182$\mathrm{PDK} 4$ axis regulates lung tumorigenesis by modulating pyruvate dehydrogenase and lipogenesis. Oncogene 36, 989-998. doi: 10.1038/onc.2016.265

Li, Z., Peng, Y., Li, J., Chen, Z., Chen, F., Tu, J., et al. (2020). N(6)-methyladenosine regulates glycolysis of cancer cells through PDK4. Nat. Commun. 11:2578.

Lincet, H., and Icard, P. (2015). How do glycolytic enzymes favour cancer cell proliferation by nonmetabolic functions? Oncogene 34, 3751-3759. doi: 10 . 1038/onc. 2014.320

Liu, L. X., Rowe, G. C., Yang, S., Li, J., Damilano, F., Chan, M. C., et al. (2017). PDK4 inhibits cardiac pyruvate oxidation in late pregnancy. Circ. Res. 121, 1370-1378. doi: 10.1161/circresaha.117.311456

Mengual, L., Ars, E., Lozano, J. J., Burset, M., Izquierdo, L., Ingelmo-Torres, M., et al. (2014). Gene expression profiles in prostate cancer: identification of candidate non-invasive diagnostic markers. Actas Urol. Esp. 38, 143-149. doi: 10.1016/j.acuroe.2013.12.002

Michelakis, E. D., Webster, L., and Mackey, J. R. (2008). Dichloroacetate (DCA) as a potential metabolic-targeting therapy for cancer. Br. J. Cancer 99, 989-994. doi: $10.1038 /$ sj.bjc.6604554

Pelicano, H., Martin, D. S., Xu, R. H., and Huang, P. (2006). Glycolysis inhibition for anticancer treatment. Oncogene 25, 4633-4646. doi: 10.1038/sj.onc.1209597

Pettersen, I. K. N., Tusubira, D., Ashrafi, H., Dyrstad, S. E., Hansen, L., Liu, X. Z., et al. (2019). Upregulated PDK4 expression is a sensitive marker of increased fatty acid oxidation. Mitochondrion 49, 97-110. doi: 10.1016/j.mito.2019.07.009

Shi, X., Shao, X., Liu, B., Lv, M., Pandey, P., Guo, C., et al. (2020). Genomewide screening of functional long noncoding RNAs in the epicardial adipose tissues of atrial fibrillation. Biochim. Biophys. Acta Mol. Basis Dis. 1866:165757. doi: $10.1016 /$ j.bbadis. 2020.165757

Spencer, N. Y., and Stanton, R. C. (2019). The warburg effect, lactate, and nearly a century of trying to cure cancer. Semin. Nephrol. 39, 380-393. doi: 10.1016/j. semnephrol.2019.04.007

Sun, S., Liu, J., Zhao, M., Han, Y., Chen, P., Mo, Q., et al. (2017). Loss of the novel mitochondrial protein FAM210B promotes metastasis via PDK4-dependent metabolic reprogramming. Cell Death Dis. 8:e2870. doi: 10.1038/cddis.2017. 273
Sun, Y., Daemen, A., Hatzivassiliou, G., Arnott, D., Wilson, C., Zhuang, G., et al. (2014). Metabolic and transcriptional profiling reveals pyruvate dehydrogenase kinase 4 as a mediator of epithelial-mesenchymal transition and drug resistance in tumor cells. Cancer Metab. 2:20. doi: 10.1186/2049-3002-2-20

Sun, Y., Gu, C., Shi, J., Fang, W., Luo, Q., Hu, D., et al. (2017). Reconstruction of mediastinal vessels for invasive thymoma: a retrospective analysis of 25 cases. J. Thorac. Dis. 9, 725-733. doi: 10.21037/jtd.2017.03.03

Sutendra, G., and Michelakis, E. D. (2013). Pyruvate dehydrogenase kinase as a novel therapeutic target in oncology. Front. Oncol. 3:38. doi: 10.3389/fonc.2013. 00038

Sutendra, G., Dromparis, P., Kinnaird, A., Stenson, T. H., Haromy, A., Parker, J. M., et al. (2013). Mitochondrial activation by inhibition of PDKII suppresses HIFla signaling and angiogenesis in cancer. Oncogene 32, 1638-1650. doi: 10.1038/onc.2012.198

Van Cutsem, E., Sagaert, X., Topal, B., Haustermans, K., and Prenen, H. (2016). Gastric cancer. Lancet 388, 2654-2664.

Wang, J., Qian, Y., and Gao, M. (2019). Overexpression of PDK4 is associated with cell proliferation, drug resistance and poor prognosis in ovarian cancer. Cancer Manag. Res. 11, 251-262. doi: 10.2147/cmar.s185015

Yang, C., and Li, B. (2019). Maintaining proton homeostasis is an essential role of the Warburg effect in proliferating cells. Cancer Biol. Med. 16, 391-395.

Yang, C., Wang, S., Ruan, H., Li, B., Cheng, Z., He, J., et al. (2019). Downregulation of PDK4 increases lipogenesis and associates with poor prognosis in hepatocellular carcinoma. J. Cancer 10, 918-926. doi: 10.7150/ jca. 27226

Yu, L., Chen, X., Sun, X., Wang, L., and Chen, S. (2017). The glycolytic switch in tumors: how many players are involved? J. Cancer 8, 3430-3440. doi: 10.7150/ jca. 21125

Zhang, L., Shi, X., Gu, C., Chen, B., Wang, M., Yu, Y., et al. (2020). Identification of cell-to-cell interactions by ligand-receptor pairs in human fetal heart. Biochim. Biophys. Acta Mol. Basis Dis. 1866:165917. doi: 10.1016/j.bbadis.2020.16 5917

Zhang, Y., Ma, K., Sadana, P., Chowdhury, F., Gaillard, S., Wang, F., et al. (2006). Estrogen-related receptors stimulate pyruvate dehydrogenase kinase isoform 4 gene expression. J. Biol. Chem. 281, 39897-39906. doi: 10.1074/jbc.m60865 7200

Zheng, J. (2012). Energy metabolism of cancer: glycolysis versus oxidative phosphorylation (Review). Oncol. Lett. 4, 1151-1157. doi: 10.3892/ol.2012.928

Zhong, H., De Marzo, A. M., Laughner, E., Lim, M., Hilton, D. A., Zagzag, D., et al. (1999). Overexpression of hypoxia-inducible factor lalpha in common human cancers and their metastases. Cancer Res. 59, 5830-5835.

Conflict of Interest: The authors declare that the research was conducted in the absence of any commercial or financial relationships that could be construed as a potential conflict of interest.

Copyright (C) 2021 Liu, Zhang and Suo. This is an open-access article distributed under the terms of the Creative Commons Attribution License (CC BY). The use, distribution or reproduction in other forums is permitted, provided the original author(s) and the copyright owner(s) are credited and that the original publication in this journal is cited, in accordance with accepted academic practice. No use, distribution or reproduction is permitted which does not comply with these terms. 